\title{
eXtreme Adaptive Optics Planet Imager: overview and status
}

Bruce A. Macintosh, Brian Bauman, Julia Wilhelmsen Evans, James R. Graham, Christopher Lockwood, et al.

Bruce A. Macintosh, Brian Bauman, Julia Wilhelmsen Evans, James R. Graham, Christopher Lockwood, Lisa Poyneer, Daren Dillon, Don T. Gavel, Joseph J. Green, James P. Lloyd, Russell B Makidon, Scot Olivier, Dave Palmer, Marshall D. Perrin, Scott Severson, Andrew I. Sheinis, Anand Sivaramakrishnan, Gary Sommargren, Remi Soummer, Mitchell Troy, J. Kent Wallace, Edward Wishnow, "eXtreme Adaptive Optics Planet Imager: overview and status," Proc. SPIE 5490, Advancements in Adaptive Optics, (25 October 2004); doi: 10.1117/12.552188

Event: SPIE Astronomical Telescopes + Instrumentation, 2004, Glasgow, United Kingdom 


\title{
eXtreme Adaptive Optics Planet Imager: Overview and status
}

\author{
Bruce Macintosh $^{* a b}$, Brian Bauman ${ }^{\text {ab }}$, Julia Wilhelmsen Evans ${ }^{\text {ab }}$, James Graham ${ }^{\text {ac }}$, Christopher \\ Lockwood $^{\mathrm{d}}$, Lisa Poyneer ${ }^{\mathrm{ab}}$, Daren Dillon ${ }^{\mathrm{d}}$, Don Gavel ${ }^{\mathrm{ad}}$, Joe Green ${ }^{\mathrm{e}}$, James Lloyd ${ }^{\text {af }}$, Russ \\ Makidon $^{\mathrm{ag}}$, Scot Olivier ${ }^{\mathrm{ab}}$, Dave Palmer ${ }^{\mathrm{ab}}$, Marshall Perrin ${ }^{\mathrm{ac}}$, Scott Severson ${ }^{\mathrm{ad}}$, Andrew Sheinis ${ }^{\mathrm{ad}}$, \\ Anand Sivaramakrishnan ${ }^{\mathrm{ag}}$, Gary Sommargren ${ }^{\mathrm{ab}}$, Remi Soumer ${ }^{\mathrm{ag}}$, Mitchell Troy ${ }^{\mathrm{ae}}$, Kent Wallace ${ }^{\mathrm{ae}}$, \\ Edward Wishnow ${ }^{\mathrm{b}}$ \\ ${ }^{a}$ NSF Center for Adaptive Optics \\ ${ }^{\mathrm{b}}$ Lawrence Livermore National Laboratory, 7000 East Ave., Livermore, CA 94551 \\ ${ }^{c}$ University of California, 601 Campbell Hall, Berkeley, CA 94720 \\ ${ }^{\mathrm{d}}$ University of California, 1156 High Street, Santa Cruz, CA 95064 \\ J Jet Propulsion Laboratory, 4800 Oak Grove Drive, Pasadena, CA 91109 \\ ${ }^{\mathrm{f}}$ California Institute of Technology, 210 East California Blvd, Pasadena, CA 91125 \\ ${ }^{\mathrm{g}}$ Space Telescope Science Institute, 3500 San Martin Drive, Baltimore, MD 21218
}

\begin{abstract}
As adaptive optics (AO) matures, it becomes possible to envision AO systems oriented towards specific important scientific goals rather than general-purpose systems. One such goal for the next decade is the direct imaging detection of extrasolar planets. An "extreme" adaptive optics (ExAO) system optimized for extrasolar planet detection will have very high actuator counts and rapid update rates - designed for observations of bright stars - and will require exquisite internal calibration at the nanometer level. In addition to extrasolar planet detection, such a system will be capable of characterizing dust disks around young or mature stars, outflows from evolved stars, and high Strehl ratio imaging even at visible wavelengths.
\end{abstract}

The NSF Center for Adaptive Optics has carried out a detailed conceptual design study for such an instrument, dubbed the eXtreme Adaptive Optics Planet Imager or XAOPI. XAOPI is a 4096-actuator AO system, notionally for the Keck telescope, capable of achieving contrast ratios $>10^{7}$ at angular separations of $0.2-1$ ". ExAO system performance analysis is quite different than conventional AO systems - the spatial and temporal frequency content of wavefront error sources is as critical as their magnitude. We present here an overview of the XAOPI project, and an error budget highlighting the key areas determining achievable contrast. The most challenging requirement is for residual static errors to be less than $2 \mathrm{~nm}$ over the controlled range of spatial frequencies. If this can be achieved, direct imaging of extrasolar planets will be feasible within this decade.

Keywords: Adaptive optics, extrasolar planets, coronagraphs, MEMS

\section{INTRODUCTION}

Over the past decade, more than one hundred extrasolar planets have been detected indirectly, through radial-velocity variations in their parent stars or more recently through occultations. One of the next significant steps in our understanding of other solar systems may be direct detection of the giant planets in these systems. Imaging extrasolar planets will open up new areas of planetary phase space, particularly planets in 5-50 AU orbits, and allow characterization of planet properties and evolution. Ground-based direct detection is extremely challenging due to the large phase errors induced by the Earth's atmosphere, but is potentially within reach of dedicated ultra-high-contrast "extreme" adaptive optics systems. Such systems must be carefully designed, however, to avoid systematic effects that could swamp planetary signals, and to insure that the system has a significant scientific reach. For example, achieving a contrast of $10^{9}$ - sufficient to detect mature Jupiter-like planets - is likely to be possible around only a handful of the brightest stars, too small a target sample to justify a dedicated AO system.

\footnotetext{
*bmac@igpp.llnl.gov
} 
We have carried out a design study for an ExAO system with broad scientific reach, called the eXtreme Adaptive Optics Planet Imager (XAOPI.) XAOPI has been designed primarily for the 10-m W.M. Keck I or II telescopes, under the auspices of the NSF Center for Adaptive Optics. XAOPI is optimized to achieve contrast - defined as the brightness ratio between a detectable planet and its parent star - of $10^{7}$ for targets with $\mathrm{m}_{\mathrm{I}}<7$. This will allow detection of selfluminous Jovian planets ${ }^{1,2}$ - slightly more massive or slightly younger than Jupiter - around a significant sample of

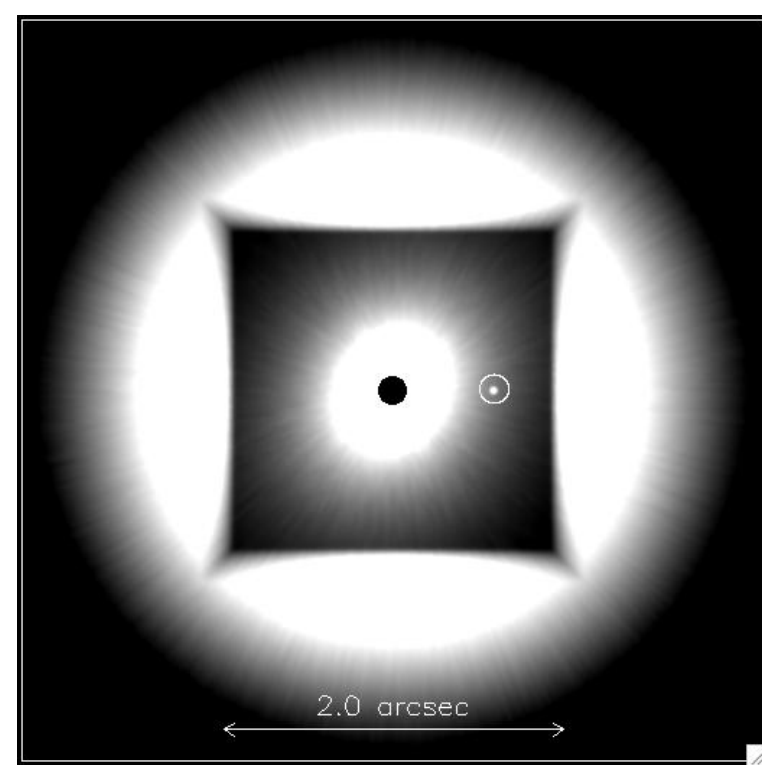

Figure 1: Simulated 450-second H-band XAOPI image of a 4 Jupiter-mass / 500 Myr planet 6 AU from a G0 star at $10 \mathrm{pc}$ distance. The characteristic square dark hole region is produced by controlling phase errors over a square region in spatial frequency space; the region is set by the square geometery of the spatially-filtered Shack-Hartmann wavefront sensor and the deformable mirror. target stars. Monte Carlo simulations based on extrapolations of the known radial-velocity distribution show that XAOPI will be able to detect $\sim 8 \%$ of planets in a typical field-star sample. Figure 1 shows a simulated XAOPI image of a nearby star with a 4 Jupiter-mass planet.

\section{SYSTEM ARCHITECTURE}

Figure 2 shows a block diagram of the XAOPI system, and Figure 3 shows the optomechanical layout.

The high-order deformable mirror (DM) will be a 4096actuator MEMS (micro-electro-mechanical systems) device. The current state of the art in MEMS DMs is the 1024-actuator continuous facesheet device from Boston Micromachines, which BM has indicated can be scaled up to 4096 actuators. Initial laboratory results with a 1024-actuator MEMS DM, including flattening to $<2 \mathrm{~nm}$ RMS over the controlled spatial frequency range, are given in a paper by Evans et $\mathrm{al}^{3}$. Since MEMS devices have a limited stroke (currently $~ 2$ microns), it is designed to operate as a post-corrector to the existing Keck AO system. The 349-actuator DM in the Keck AO system, under control of its own wavefront sensor, will remove the bulk of the atmospheric turbulence, allowing the XAOPI MEMS to provide precision correction ${ }^{4}$. The Keck AO system also provides tip/tilt and image rotation services, reducing the size and complexity of XAOPI.

The primary wavefront sensor for XAOPI will be a spatially-filtered Shack-Hartmann wavefront sensor (SFWFS). As discussed in section 4.1 and in more detail in a recent paper by Poyneer and Macintosh ${ }^{5}$, a square hole of size $\lambda / \mathrm{d}=0.9$ arcseconds can significantly reduce aliasing of unmeasurable and uncorrectable spatial frequencies into the controllable range, producing a PSF with a well-defined "dark hole" region for planet detection. Spatial filtering operates most effectively at high Strehl ratios; hence the sensor will operate at the longest range practical for current (CCD) detector technology, 0.7-0.9 microns. The filter size will also be adjustable, allowing initial lock with an oversized aperture. The baseline detector is a $128 \times 128$ pixel CCD operating at $2.5 \mathrm{kHz}$. This geometry (barely) allows $2 \times 2$ pixels per subaperture with no guard bands.

The XAOPI coronagraph is a self-contained warm unit, allowing a variety of science instruments to be used. A companion paper $^{6}$ discusses the coronagraph architecture, most likely a band-limited ${ }^{7}$ Lyot coronagraph. XAOPI is designed to have its own dedicated science camera, initially a simple direct 1-2.5 micron imager. Other existing Keck instruments can be fed with the same $\mathrm{f} / 15$ beam, such as the OSIRIS integral field spectrometer ${ }^{8}$. OSIRIS has too narrow a field of view to be used for extrasolar planet searches, but could be used for spectroscopic follow-up.

A key feature of XAOPI is the second "calibration" wavefront sensor, used for precision monitoring of the wavefront delivered through the coronagraph. As shown in section 4, small static wavefront errors can completely dominate the high-contrast sensitivity of a ExAO system. The calibration wavefront sensor will provide a time-averaged measurement of the wavefront at the science wavelength with high $(\sim 1 \mathrm{~nm})$ accuracy. Any average static wavefront 
offsets detected can be fed back as offsets into the main AO control loop. A separate paper ${ }^{9}$ discusses options for this wavefront sensor. In addition, a phase-shifting point-diffraction interferometer (PSDI ${ }^{10}$ ) provides an absolute measurement of the optical path from the input focus to the coronagraph input, to be used for daytime calibration.

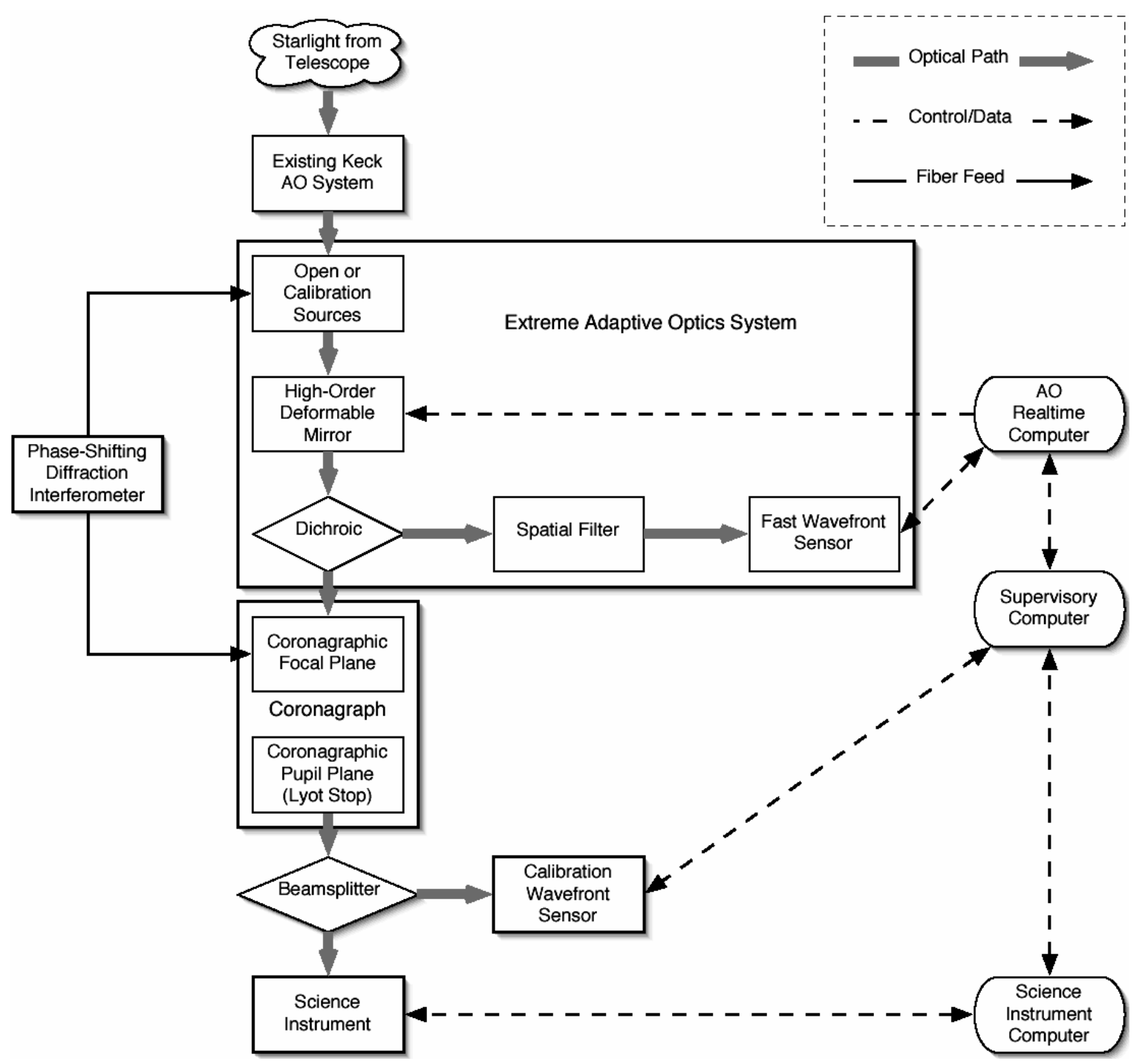

Figure 2: Block diagram of the XAOPI system.

\section{PERFORMANCE MODELLING}

The figure of merit for the error budget of an ExAO system is not the total RMS wavefront error or Strehl ratio, but the final achievable contrast. This means that we require tools - analytic or simulation - for translating different wavefront error terms into their effects on contrast. Several families of simulations have been used to predict XAOPI performance, ranging from detailed wave-optics simulations to semi-analytic error budgets (section 4.) The full optics simulations (e.g. those of the SFWFS) can be used to determine the detailed behavior of system components, but are computationally prohibitive for determining final sensitivities. Simulating one second of exposure time at twice Nyquist sampling with our current codes takes $\sim 16$ hours on a dual-processor Macintosh G5, and many effects (such as small static wavefront errors) only become apparent in hour-long exposures. We use two techniques for predicting final sensitivity. The first are Monte Carlo Fourier-domain simulations in which a series of independent phase screens are generated to represent timesteps separated by the individual speckle lifetimes rather than the AO update rate. The AO 
correction is simulated with a Fourier-domain filter, and additional error sources such as measurement noise or temporal bandwidth are modeled as additive noise at appropriate spatial frequencies and shifts in the corrected phase. These techniques produced the images shown in e.g. Figure 1. However, even this technique is too computational intensive to explore a variety of system designs, so we must turn to semi-analytic error budgets.

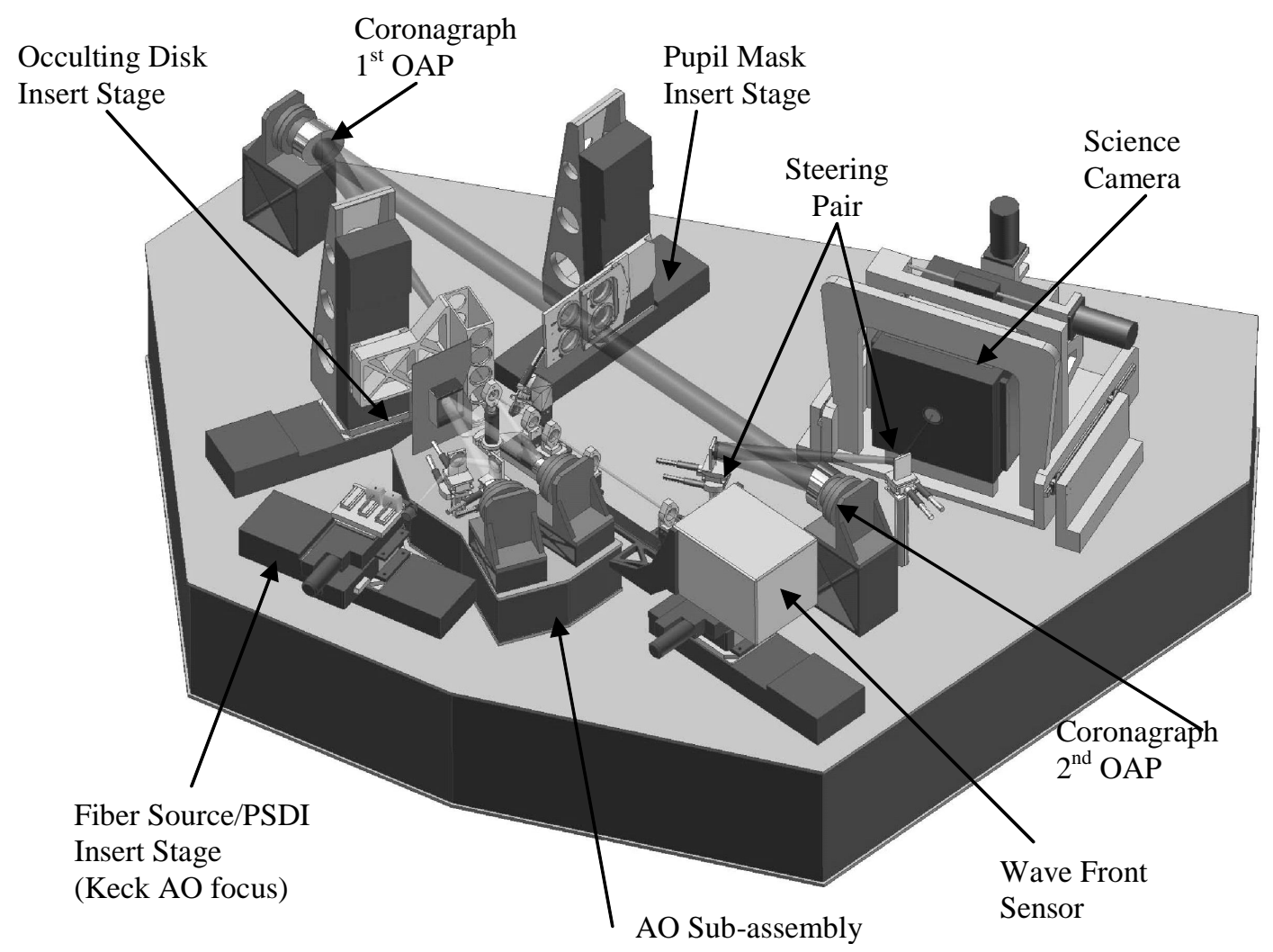

Figure 3: 3D rendering of the XAOPI optical bench.

As shown by Sivaramakrishnan et al. ${ }^{11}$, the high-contrast PSF in the high-Strehl regime can be written as the sum of a diffraction pattern term, a "pinned speckle" term (ref) which also traces the diffraction pattern, and a PSF halo term. The first two can be suppressed by a coronagraph. The third term, which dominates the wings ( $>3-5 \lambda / \mathrm{D})$ of an ExAO system with an effective coronagraph, is essentially the power spectrum of the residual phase errors. This provides a natural way to predict the long-exposure average PSF and final sensitivity to pointlike companions. Assuming there are $\mathrm{n}$ distinct wavefront error sources $\phi_{i}$ and all are uncorrelated, the total PSF intensity (normalized to unity) is given by $P(\theta)=\sum_{i}^{n} \sigma_{i}^{2} I_{i}(\theta)$ where $I_{i}(\theta)=<\left|\Phi_{i}(\theta / \lambda)\right|^{2}>/ \sigma_{i}^{2}$ is a the unity-normalized spatial power spectrum of the phase error $\phi_{i}$ and $\sigma_{i}$ is the magnitude of the corresponding phase error in radians. A phase error of spatial frequency $\theta / \lambda$ in cycles per meter $(D \theta / \lambda$ in cycles per pupil) will scatter light to an angular radius $\theta$. This leads to a useful insight: in order to detect planets at radii between 0.2 and 1 arscecond at $\mathrm{H}$ band, we need to control phase errors between $\sim 3$ cycles per pupil and $\sim 30$ cycles per pupil. Lower frequency errors will primarily rearrange light under the coronagraph occulting stop (though the details of how light leaks through the coronagraph are complex for different designs) while higher frequencies scatter light to large radii. Reducing the errors at mid frequencies results in a PSF with a "dark hole" ${ }^{, 12}$ or basin in which planets can be detected. 


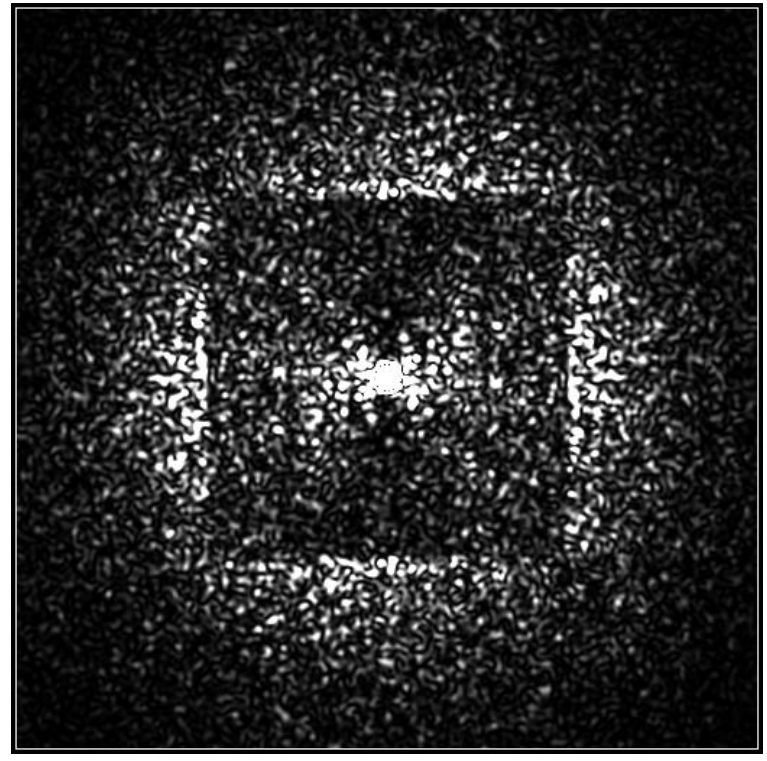

Figure 4: Instantaneous monochromatic PSF showing speckles

$N(\theta, t)=\sum_{i}^{n} \sigma_{i}^{2} I_{i}(\theta)\left(\frac{t d e c_{i}}{t}\right)^{1 / 2}$
Instantaneously, the PSF is completely broken up into speckles (Figure 4)- and these speckles are the main source of noise in an attempt to detect a pointlike object such as a planet. The noise as a function of radius for a single PSF noise source in monochromatic light is given by $\sigma_{i}^{2} I_{i}(\theta)$ - the noise is roughly equal to the intensity. (In broadband light, an additional term appears decreasing the noise due to the elongation of the speckles; for clarity we will omit this term in this section, though it has been included in our error budget in section 4.) Over a long integration, multiple realizations of the speckle pattern will act to smooth the PSF. We express this by assigning each error source a characteristic speckle decorrelation timescale $t d e c_{i}$; in an integration time $t>t d e c_{i}$, the final noise for a single source will be given by $\sigma_{i}^{2} I_{i}(\theta)\left(\frac{t d e c_{i}}{t}\right)^{1 / 2}$

If multiple error sources are present, Sivaramakrishnan et al (2002) show that each decorrelates independently, and the total noise in the final image is given by

This means that error sources with rapid decorrelation, such as the random measurement noise of the AO system, are much less significant than errors that decorrelate slowly, such as the atmospheric fitting and bandwidth terms. Errors that do not decorrelate, such as quasi-static optical errors, are the worst of all; as shown by Sivaramakrishnan et al, in an extremely long exposure with both random and static errors, the PSF approaches the noise floor given by the static errors only - the PSF becomes a smooth halo with imprinted on it a speckle pattern equivalent to that given only by the static errors. Figure 8 illustrates this and shows the severe effect of even small static PSF errors.

\section{ERROR BUDGET AND PSF COMPONENTS}

Using this formalism, we can produce an error budget for XAOPI that will allow us to not only predict Strehl ratio but also estimate final contrast at a given radius, as in Table 1. In the following sections we will briefly discuss the properties of each error source and in several cases illustrate the PSF that would occur if that were the only error source; as discussed above, the final long-exposure PSF will be given by the sum of these separate PSFs, at radii where the diffraction component is negligible.

We divide each error source into three spatial frequency components: low frequencies ( $<4$ cycles/pupil), corresponding to wavefront error sources that primarily scatter light beneath the occulting spot of the coronagraph; high frequencies (>30 cycles per pupil), which scatter light into the wings of the PSF at large angles; and the crucial mid-frequency range that corresponds to the dark hole in which we wish to detect planets. For each error source, we present the corresponding scattered-light intensity at a radius of 0.4 arcseconds, and the resulting speckle noise in a 3600-second exposure. (Note that since speckle noise occurs from the intensity of the PSF, which is already a squared quantity, the speckle noises add linearly rather than in quadrature.) The contribution to the final noise due to photon noise is also listed. The predicted detectable point source contrast $-7.5 \times 10^{-7}$ at 0.4 arcseconds in a one-hour integration - agrees within a factor of two with more detailed simulations. Figure 5 shows the contributions of each error source graphically. For comparison, Figure 6 shows a similar chart for a typical non-extreme AO system such as the current Keck AO. Note that we do not assume any PSF subtraction, either through observations of reference stars or through multi-wavelength imaging ${ }^{13,14}$; this is a conservative error budget for direct broadband imaging. If quasi-static PSF components can be removed through some post-processing, requirements on static error sources could be relaxed. Key individual error sources are discussed in the following sections. 


\begin{tabular}{|l|l|l|l|l|r|r|}
\hline WFE source & $\begin{array}{l}\text { low freq. } \\
\text { WFE } \\
\text { (nm) }\end{array}$ & $\begin{array}{l}\text { mid freq. } \\
\text { WFE } \\
(\mathrm{nm})\end{array}$ & $\begin{array}{l}\text { high freq. } \\
\text { WFE } \\
(\mathrm{nm})\end{array}$ & $\begin{array}{l}\text { lall freq } \\
\text { WFE } \\
(\mathrm{nm})\end{array}$ & $\begin{array}{l}\text { PSF intensity } \\
\text { l.4 arcsec }\end{array}$ & $\begin{array}{l}\text { PSF noise } \\
0.4 \text { arcsec } \\
3600 \text { seconds }\end{array}$ \\
\hline Atmosphere fitting & 0.0 & 2.0 & 39.8 & 39.9 & $7.6 \times 10^{-8}$ & $9.6 \times 10^{-11}$ \\
\hline Telescope primary/secondary & 0.0 & 1.1 & 22.0 & 22.0 & $2.3 \times 10^{-8}$ & $2.5 \times 10^{-9}$ \\
\hline Telescope vibration & 0.0 & 0.6 & 11.0 & 11.0 & $5.7 \times 10^{-9}$ & $1.6 \times 10^{-11}$ \\
\hline Initial calibration & 5.0 & 1.0 & 0.0 & 5.1 & $1.9 \times 10^{-8}$ & $2.1 \times 10^{-9}$ \\
\hline Atmospheric bandwidth & 16.4 & 17.4 & 12.1 & 26.4 & $3.2 \times 10^{-6}$ & $4.0 \times 10^{-9}$ \\
\hline WFS measurement & 0.0 & 11.3 & 0.0 & 11.3 & $2.4 \times 10^{-6}$ & $5.6 \times 10^{-10}$ \\
\hline Uncorrectable internal errors & 0.0 & 0.0 & 30.0 & 30.0 & 0.0 & 0.0 \\
\hline Quad cell gain changes & 0.4 & 0.2 & 0.0 & 0.4 & $7.6 \times 10^{-10}$ & $8.2 \times 10^{-11}$ \\
\hline System Flexure & 10.0 & 1.0 & 0.0 & 10.0 & $1.9 \times 10^{-8}$ & $2.1 \times 10^{-9}$ \\
\hline Post-coronagraph aberrations & 30.0 & 5.0 & 5.0 & 30.8 & $2.7 \times 10^{-8}$ & $3.0 \times 10^{-9}$ \\
\hline Photon noise & & & & & & $9.4 \times 10^{-10}$ \\
\hline Total & $\mathbf{1 9 . 8}$ & $\mathbf{2 0 . 9}$ & $\mathbf{5 6 . 9}$ & $\mathbf{6 3 . 8}$ & $\mathbf{5 . 7 \times 1 0 ^ { - 6 }}$ & $\mathbf{1 . 5 \times 1 0 ^ { - 8 }}$ \\
\hline
\end{tabular}

Table 1: XAOPI error budget for a one-hour observation. Target $\mathrm{m}_{\mathrm{I}}=4, \mathrm{r}_{0}=20 \mathrm{~cm}$ at $500 \mathrm{~nm}$, and tau $\mathrm{u}_{0}=0.1 \mathrm{~seconds}$. See text for a discussion of the columns. Science wavelength is 1.65 microns ( $\mathrm{H}$ band.) PSF intensity and PSF noise are normalized with respect to the peak intensity of the coronagraphic PSF, so that a PSF noise of $1.5 \times 10^{-8}$ would represent a 5 -sigma detection of a companion with a contrast relative to its primary of $7.5 \times 10^{-7}$.

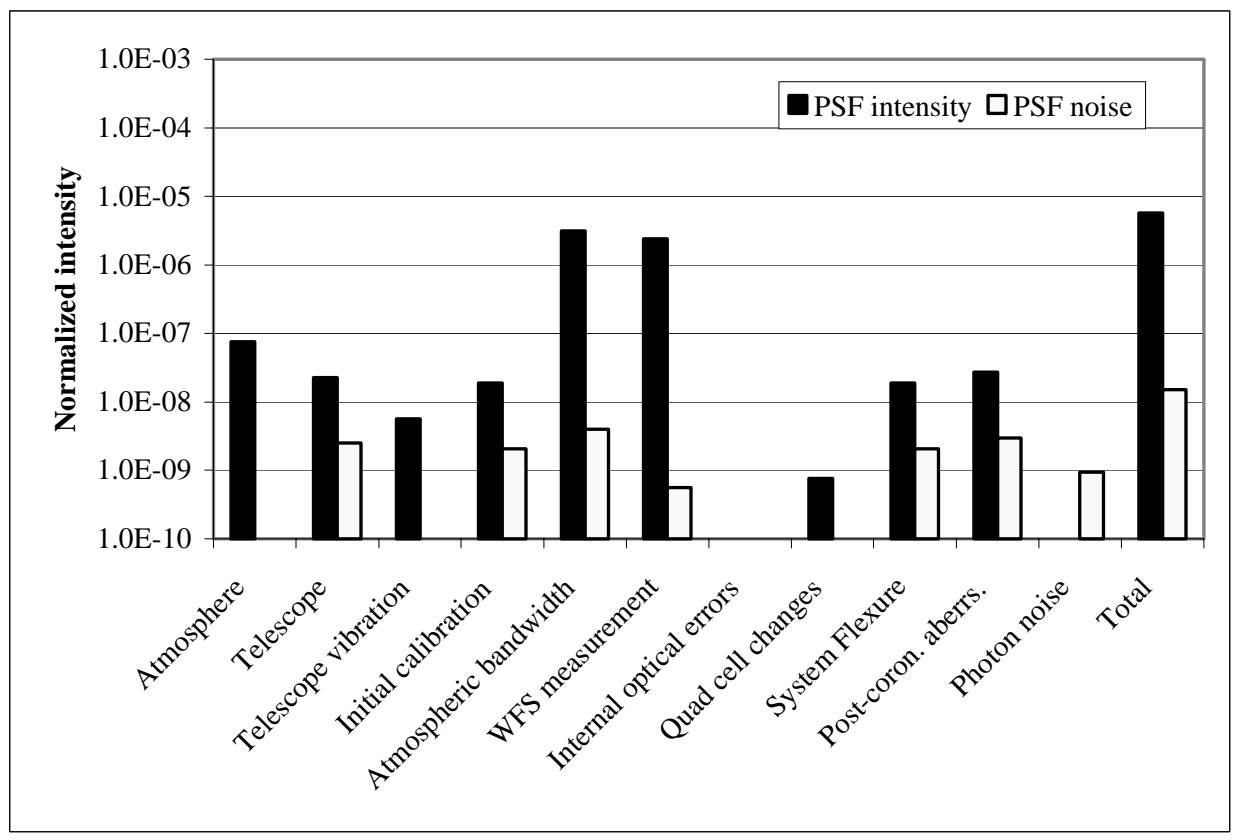

Figure 5: Contributions of each separate error source to the final PSF intensity (solid bars) and PSF noise (light bars) at 0.4 arcseconds separation in a one-hour exposure. 


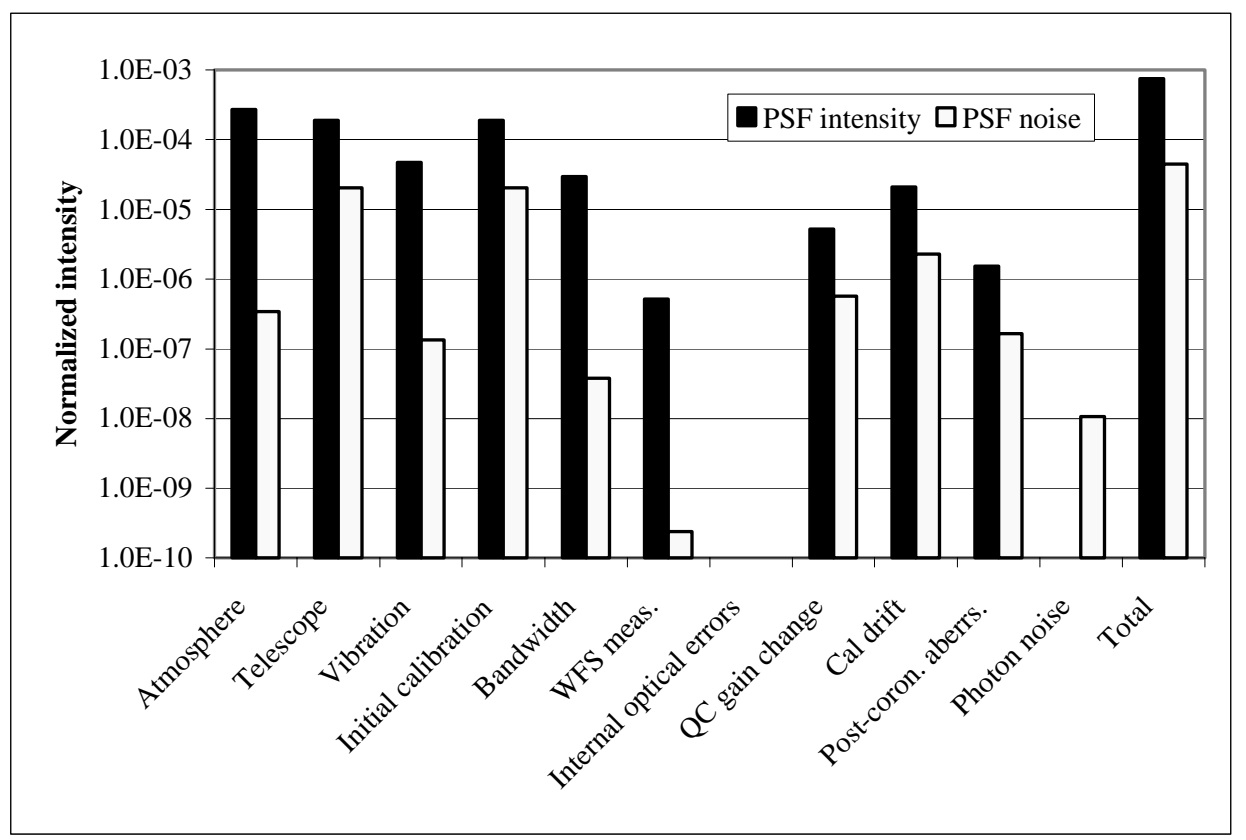

Figure 6: Contributions of various error sources for a generic current-generation AO system on the Keck telescope. The predicted contrast agrees roughly with current observations.

\subsection{Atmospheric errors}

Classic atmospheric fitting error - i.e., the portion of the atmospheric phase errors that cannot be corrected by the deformable mirror - is almost exclusively a high-frequency error source; a well-behaved DM can reproduce the bandlimited components of almost any wavefront. This produces a PSF similar to the left portion of Figure 7, bright only outside the AO control region with a small amount of error close to the star caused by fourth-order terms in the PSF expansion. Of course, most AO systems do not produce PSFs of this shape, due to aliasing effects ${ }^{5}$, resulting in PSFs similar to the right portion of figure 7, where the dark hole is filled in. XAOPI will use a spatially-filtered wavefront sensor to block aliasing; at high Strehl ratios this can reduce the amount of mid-frequency power by a factor of 100 or more ${ }^{5}$. This produces a significant increase in sensitivity, as speckles due to atmospheric fitting and aliasing errors are long-lived (tdec $=0.2-0.4$ seconds for a $10-\mathrm{m}$ telescope).

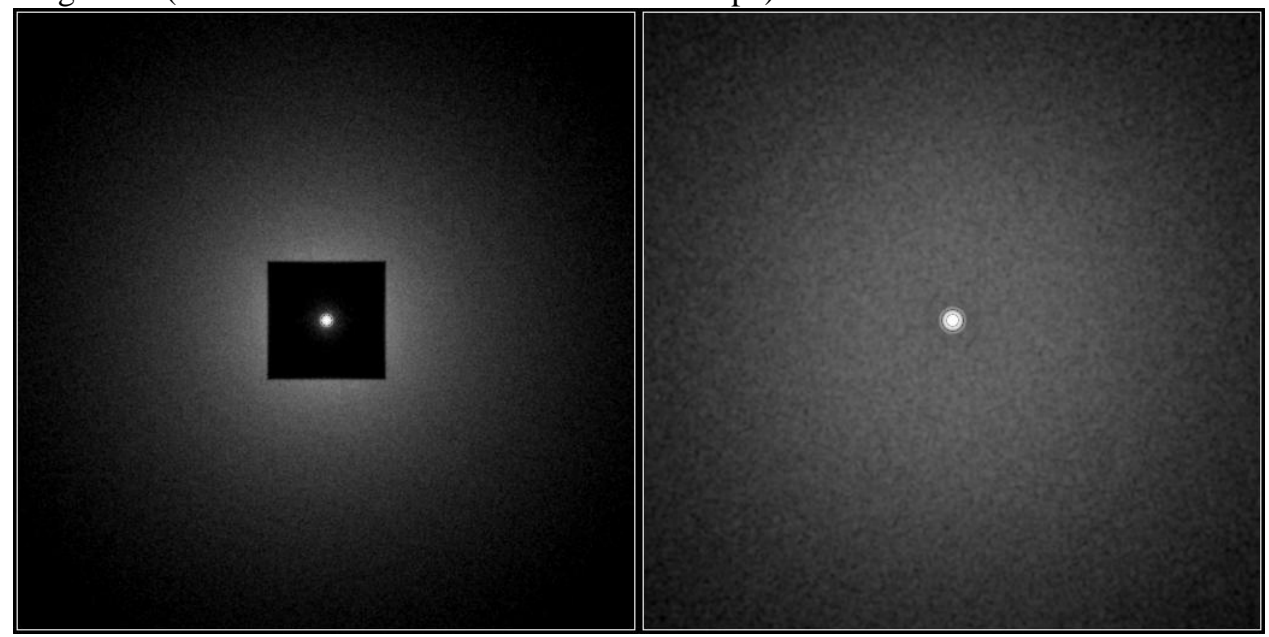

Figure 7: Simulated PSFs due to atmospheric fitting error (left) and fitting + aliasing errors (right.) Five second exposure monochromatic PSF with diffraction suppressed by pupil apodization. 


\subsection{Telescope static and vibration errors}

For a segmented telescope such as Keck, uncorrectable telescope primary mirror errors are a significant concern. Lowfrequency phase errors in individual segments and segment piston/tip/tilt errors create phase discontinuities at the edges of segments. While a continuous deformable mirror cannot perfectly reproduce these discontinuities, it can take a shape that reproduces the mid-frequency components of a phase discontinuity. See Poyneer and Macintosh $2004^{5}$ for discussion and detailed simulation results. Residual errors due to the telescope are assumed to be essentially static, smoothing out only as the telescope pupil rotates with respect to the sky, $\sim 1000$ seconds for small field angles at moderate elevations.

\subsection{Initial calibration and residual static errors}

The XAOPI optical design goal requires $<25 \mathrm{~nm}$ of non-common path optical errors between the wavefront sensor and the coronagraph input - challenging but not impossible. However, even this small amount would completely dominate final contrast sensitivity if not removed through calibration. Figure 8 illustrates the effect of small wavefront errors on final contrast.

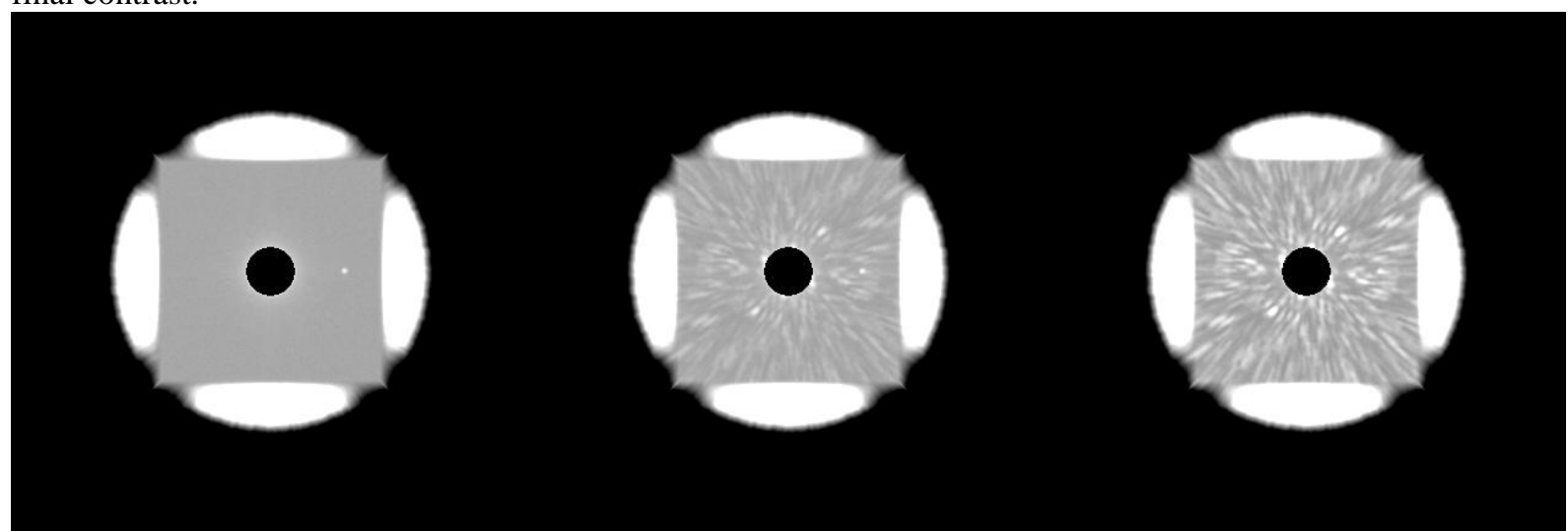

Figure 8: Three simulated 15 minute ExAO images similar to Figure 1, with 0 (left), 2, and $4 \mathrm{~nm}$ RMS random static wavefront error (uniformly distributed in spatial frequencies out to the AO cutoff). This simulation was carried out with low winds $(<5 \mathrm{~m} / \mathrm{s})$ to reduce the effect of atmospheric bandwidth errors.

Even if PSF subtraction is not used, appropriate observing modes can smooth out some of these speckles; in particular, if the system image rotator is operated to keep the telescope pupil fixed on the AO system rather than keeping the sky fixed, short-exposure images can be derotated and combined to cause both errors from the telescope and the AO bench to smooth out.

Achieving these error levels will be extremely challenging, but they are an order of magnitude less than those required for e.g. the Terrestrial Planet Finder mission. A companion paper discusses our approach to achieving and maintaining calibration, and we have demonstrated $<2 \mathrm{~nm}$ RMS wavefront error with both a flat mirror and a 1024-actuator MEMS deformable mirror on a simple ExAO testbed, with corresponding contrast $>10^{7}$.

\subsection{Atmospheric temporal bandwidth errors}

One of the two main sources of scattered light within the dark hole is the finite temporal bandwidth of the AO system its inability to keep up with the moving and evolving atmospheric turbulence. Figure 9 shows the corresponding PSF for a single Taylor screen, this has a characteristic "butterfly" shape, though of course multiple layers and deviations from frozen-flow will cause the PSF to become more symmetric. In Figure 1 this is the main source of scattered light close to the star. As with other atmospheric errors, this produces moderately long-lived (0.2-0.4 second) speckles, so that even with XAOPI's aggressive $2500 \mathrm{~Hz}$ sample rate, this remains the dominant external term in the final contrast budget. Predictive or other advanced controllers could help to reduce this effect, but such controllers have yet to be implemented with practical algorithms or tested, so our performance models assume only a simple control loop.

\subsection{Wavefront sensor measurement noise}

The second major source of scattered light inside the AO control radius is wavefront errors injected by the AO system itself due to finite SNR in the wavefront sensor. By definition, this is a source only of errors within the controlled range 
of spatial frequencies, and can be significant for dim target stars. Fortunately, since these errors are uncorrelated from measurement to measurement, they produce speckles that decorrelate rapidly - compare the smoothness of the righthand image in Figure 9 to the left-hand image. In a closed-loop controller this decorrelation does not take place at the full update rate of the AO system; we have assumed a tdec $=1 / f_{b}$ where $f_{b}$ is the $3 \mathrm{db}$ bandwidth of the controller, but detailed closed-loop AO simulations will be needed to determine the exact evolution of the speckle pattern.

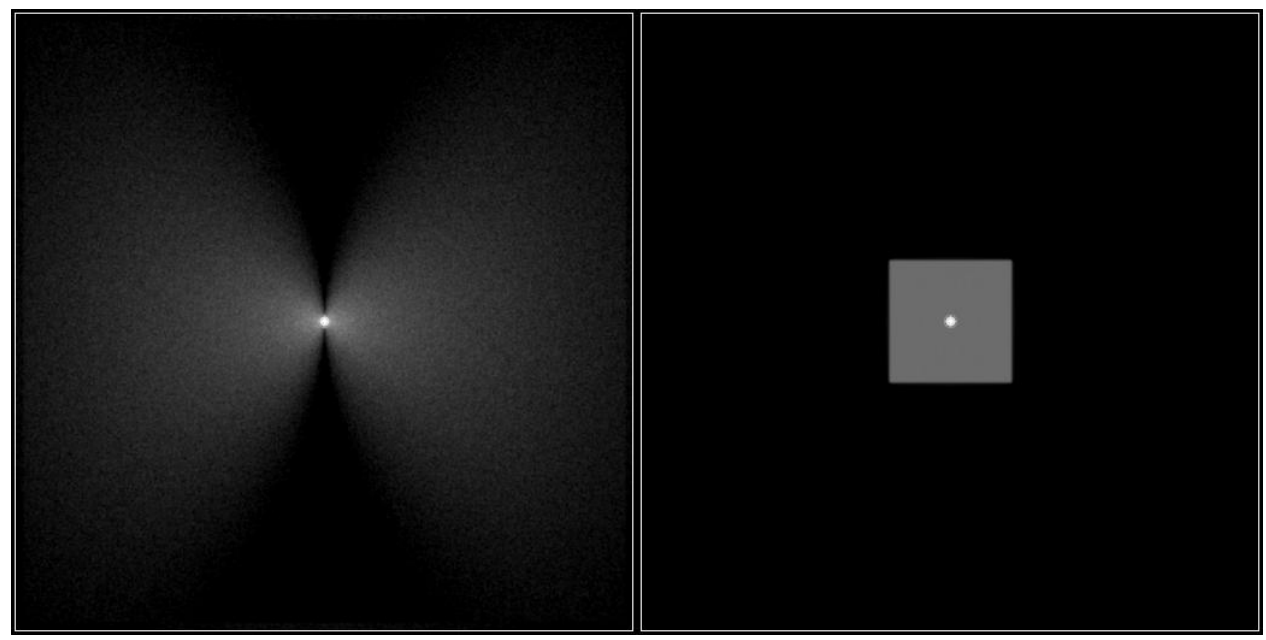

Figure 9: Left: Simulated PSF due to temporal bandwidth errors with the wind moving left to right. Right: Simulated PSF due to wavefront measurement noise for a spatially-filtered direct-phase measurement wavefront sensor. Five second exposure monochromatic PSF with diffraction suppressed by pupil apodization.

\subsection{Quad cell gain changes}

Many AO systems, including XAOPI, use Shack-Hartmann wavefront sensors in a quad cell mode with only $2 \times 2$ pixels per subaperture. Although this is the most efficient in terms of detector real estate and readout noise, it results in a sensor whose gain is a function of the size of the Shack-Hartmann spot. The spot size, of course, can change due to changes in $r_{0}$ or even random realizations of the wavefront error across a subaperture at a given $r_{0}$. These changes in gain can have two detrimental effects. First, imperfect knowledge of the gain can lead to decreased bandwidth in the AO system. Second, and more significantly, if the AO system is attempting to maintain a non-flat residual wavefront offset from flat on the wavefront sensor, e.g. due to non-common-path errors, the mis-estimation of the gain will cause the average centroids to be offset from the desired wavefront, over- or under-correcting the non-common-path errors. Several techniques have been suggested to overcome this, including the modulation of the wavefront by the AO system with a known error signal at a rate beyond the $\mathrm{AO}$ control bandwidth ${ }^{15}$, but it is unclear if these are precise enough for our error budget requirements.
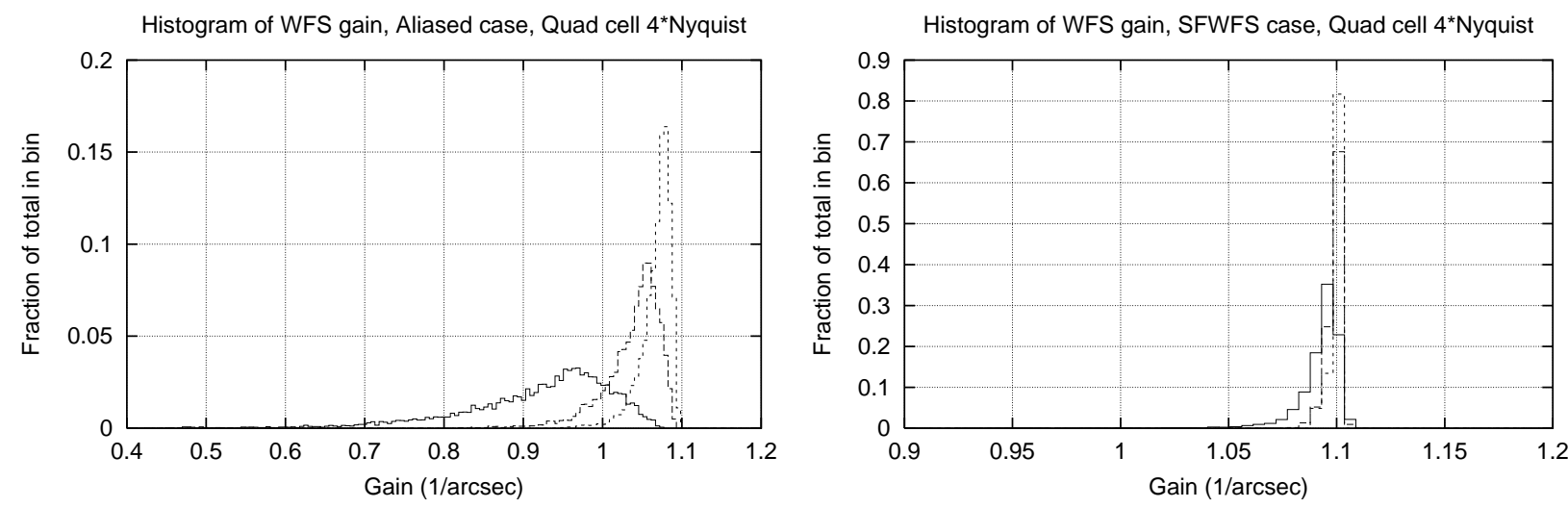

$$
\mathrm{r} 0=10 \mathrm{~cm} \longrightarrow \quad \mathrm{r} 0=20 \mathrm{~cm}-\cdots=30 \mathrm{~cm} \cdots \ldots .
$$

$$
\mathrm{r} 0=10 \mathrm{~cm} \longrightarrow \mathrm{r} 0=20 \mathrm{~cm}-\cdots \quad \mathrm{r} 0=30 \mathrm{~cm} \cdots \cdots
$$

Figure 10: Histogram of quad-cell gains for Shack-Hartmann wavefront sensors, $\mathrm{d}=16 \mathrm{~cm}$, with (left) and without (right) a spatial filter. Note the change in axis scales. 
Fortunately, an additional advantage of the XAOPI spatially-filtered wavefront sensor is that it acts to suppress these changes in gain. Figure 10 shows histograms of the gain at three different $r_{0}$ values for a conventional and spatiallyfiltered WFS. The SFWFS values show extremely little scatter or $r_{0}$ dependence, nearly matching the values for a perfect diffraction-limited subaperture. This is of course due to the removal of high spatial-frequency wavefront errors; this ensures that the wavefront across a given subaperture consists primarily of tip and tilt components. As a result, the WFS gain can be predicted with considerable accuracy even without an estimate of $r_{0}$.

\subsection{Post-coronagraph errors}

Errors after the coronagraph occulting spot are often neglected in ExAO modeling, since the coronagraph spot blocks a significant fraction of the light of the star. These can still be significant; to first order, the light scattered after the coronagraph is given by the light scattered before the coronagraph scaled by (1-S) where $S$ is the Strehl ratio of the system up to the coronagraph spot. Since the pupil after the coronagraph is not uniformly illuminated, correcting for both pre- and post-coronagraph errors with the deformable mirror is challenging, especially in white light, so we have assumed that these errors cannot be corrected and placed a requirement on post-coronagraph optics as given in Table 1.

\section{CONCLUSIONS AND STATUS}

The XAOPI project has lead to a detailed strawman design for a planet-finding ExAO system, based not simply on maximizing the Strehl ratio but on a detailed analysis of the final PSF. We have carried out simulations and analytic calculations to predict the final sensitivity and identify risk areas; the most challenging aspect of the design is the static wavefront error requirement of $<1-2 \mathrm{~nm}$, but we believe we have a path to achieving this goal.

XAOPI was designed primarily for use on one of the Keck telescopes, but no funding source has been identified for the construction phase. Our group has been selected by the Gemini observatory to carry out a conceptual design study for a Gemini ExAO system, with the study to be completed by early 2005. With practical designs available for two major US telescopes, and corresponding studies being carried out for the ESO VLT, we can hope to see the first planet-finding AO systems deployed before the end of this decade, bringing direct detection of extrasolar planets into reach.

\section{ACKNOWLEDGEMENTS}

This research was performed under the auspices of the U.S. Department of Energy by the University of California, Lawrence Livermore National Laboratory under Contract W-7405-ENG-48, and also supported in part by the National Science Foundation Science and Technology Center for Adaptive Optics, managed by the University of California at Santa Cruz under cooperative agreement No. AST - 9876783. Support for this work was also provided by a grant from the Gordon and Betty Moore Foundation to the Regents of the University of California, Santa Cruz, on behalf of the Laboratory for Adaptive Optics. The content of the information does not necessarily reflect the position or the policy of the Gordon and Betty Moore Foundation, and no official endorsement should be inferred.

\section{REFERENCES}

$1 \quad$ Burrows, A. et al. 1997, Ap.J., 491, 856

2 Baraffe, I., et al. 2003, A\&A 402, 701

3 Evans, J., et al., 2004 Proc. SPIE 5490 (this volume)

$4 \quad$ Poyneer, L, and Macintosh, B., 2003 Proc. SPIE 5169, 190

$5 \quad$ Poyneer, L, and Macintosh, B., 2004 JOSA A 21, 810

6 Sivaramakrishnan, A., et al. 2004 Proc. SPIE 5490 (this volume)

$7 \quad$ Kuchner, M, and Traub, W., 2002 Ap. J. 570, 900

8 Larkin, J., et al. 2003 Proc. SPIE 4841, 1600

$9 \quad$ Wallace, K., et al. 2004 Proc. SPIE 5490 (this volume)

10 Sommargren, G.E., Phillion, D.W. ,Johnson, M.A., Nguyen, N.Q., Barty, A., Snell, F.J., Dillon, D.R., Bradsher, L.S., Phillion, D.W., Campbell, E.W., 2002, Proc. SPIE Vol. 4688, p. 316-328

11 Sivaramakrishnan, A., Lloyd, J. P., Hodge, P. E., and Macintosh, B. A. 2002, ApJ, 581, L59

12 Malbert, F., Yu, J. W., and Shao, M., 1995 PASP 107, 386

13 Racine, R., et al. 1999 PASP 111, 587 
14 Sparks, W.B and Ford, H.C 2002, ApJ, 578, 543

15 Herriot, G., et al. 2004 Proc. SPIE 5490 (this volume) 